\title{
New trends in gyrotron development
}

\author{
G.G. Denisov \\ Institute of Applied Physics, Russian Academy of Sciences, Nizhny Novgorod, Russia, den@appl.sci-nnov.ru, \\ Gycom Ltd., Nizhny Novgorod, Russia
}

\begin{abstract}
Gyro-devices provide the highest $\mathrm{CW}$ or average power of microwaves in centimeter, millimeter and submillimeter wavelength ranges and therefore they are very attractive as microwave sources for many of applications such as plasma fusion, radiolocation, ion sources, telecommunication, technology, spectroscopy and some other. In last years an essential progress in the device development was demonstrated. The paper presents State-of the Art in the device development, new demands in the parameters enhancement and possible ways to achieve the goals.
\end{abstract}

\section{Gyro-Devices. State-of-the Art}

Gyrotrons for plasma fusion installations usually operate at frequencies 40-170 GHz [1-6]. Requested output power of the tubes is about $1 \mathrm{MW}$ and pulse duration is between seconds and thousands seconds (depending on plasma machine parameters). In ITER installation there will be 24 of $170 \mathrm{GHz}$ gyrotron systems with $1 \mathrm{MW}$ microwave power each. ITER requirements include also high efficiency of the gyrotrons over $50 \%$, possibility of power modulation with frequency up to $5 \mathrm{kHz}$, compatibility of the gyrotron complex with ITER control system. In May, 2015 a Russian Prototype of ITER Gyrotron System was completed and its operation was demonstrated [2-5]. The system includes gyrotron oscillator, liquid-free superconducting magnet, supplementary magnets, several electric power supplies, cooling systems control and protection systems, and other auxiliary units. The gyrotron system shows reliable operation with required parameters. In October, 2015 Final Design Review Procedure for the gyrotron system was successfully passed and in 2016 fabrication of the first serial gyrotron system was completed. Megawatt power at very long pulses (300-1000 s) was also demonstrated with gyrotron at $140 \mathrm{GHz}$ frequency for electron-cyclotron systems of EAST (China) and KSTAR (Korea) superconducting tokamaks. Megawatt power gyrotrons with moderate pulse duration from 2 to 10 seconds were developed for TCV, HL-2A, and ASDEX Upgrade tokamaks.

There are successful developments of megawatt gyrotrons $[1,6]$ by European team including industrial company Thales, by CPI (USA), by Japanese cooperation of QST/Toshiba and Tsukuba University. The point worth mentioning is that the new stellarator $\mathrm{W} 7-\mathrm{X}$ is equipped now with 9 gyrotrons capable to operate at $140 \mathrm{GHz}$ frequency and power of $0.8-1.0 \mathrm{MW}$ in 1800 second pulses.

Gyro-amplifiers are intrinsically more difficult in realization because of multi-mode microwave systems, but in recent years there is a remarkable progress in their development. At IAP/GYCOM we are developing an original concept of a gyro-TWT that is based on the use of a helically corrugated waveguide that radically changes the dispersion of the modes of a circular waveguide. The operating mode has sufficiently high and almost constant group velocity at zero axial wavenumber which enables broadband operation of the helical-waveguide gyro-TWT with minimum sensitivity to electron velocity spread. A number of experiments have proved the main theoretical predictions and advantages of gyro-TWTs of this type [7-9]. In particular the output power of about $10 \mathrm{~kW}$ and instant frequency band more than $2 \mathrm{GHz}$ were demonstrated with CW Ka-band tubes and more than $150 \mathrm{~kW}$ in pulse tubes with $10 \%$ duty factor. Several directions of research on the helical-waveguide gyro-TWT are currently under development at the IAP. Among the actual topics are the following: tubes with a high (40-50 dB) gain; devices with a new microwave system for inputting and outputting the radiation to and from a tube through one window; high-power tubes operating at the third cyclotron harmonic; tubes operating at $95 \mathrm{GHz}$ and $260 \mathrm{GHz}$ [10].

\section{New Customer Demands}

Traditional goals in gyrotron development are the power increase and the frequency increase. Future plasma machines as DEMO [11] claim for EC system higher frequency (comparing with ITER) $230 \mathrm{GHz}(170 \mathrm{GHz})$, module power increase 1.5-2.0 MW (1 MW), efficiency enhancement to value higher $60 \% \quad(50 \%)$, multifrequency operation in order to avoid wide angle scanning of wave beams in plasma. There are mutual contradictions in these listed requests, for example, the higher frequency and the higher power require bigger gyrotron cavity (higher operating mode) and this affects gyrotron efficiency. Nowadays such a combination of parameters looks unreal - similar case with ITER requirements 25 years ago.

Besides power and efficiency increase recently more requirements became more acute in aspects of oscillation spectrum control. As it was mentioned it is considered gyrotron operation at different modes with different frequencies. For one mode operation frequency stabilization is desired and frequency tuning within resonance curve is necessary. Phase and frequency locking is required to make several gyrotrons as coherent oscillators. For spectroscopy application high frequency stabilization is needed. For some cases generation of periodical sequences of ultrashort (wide spectrum) phase-coherent pulses is very useful.

\section{New Approaches and Recent Results}

There are new developments as phase locking of gyro-oscillator, stabilization of gyrotron frequency by the optimal reflection from a remote object, stabilization of gyrotron frequency by phase locking loop (PLL), and generation of ultra-short pulses in gyro-TWT with nonlinear absorber in the feedback loop [12-17]. 
A gyrotron oscillator operating efficiently at modes of both rotations was developed and tested in Proof-OfPrinciple experiment. The gyrotron operation can be switched by means of a cathode coil between two modes: co- and counter- rotating ones with respect to electron rotation. The main operating modes are $\mathrm{TE} \pm_{28.12}$ at $170 \mathrm{GHz}$ frequency. A new synthesized quasi-optical mode converter composed of 3D metal reflectors provides coupling out of the generated waves in the form of two Gaussian wave beams passing the window disk with $\pm 10^{\circ}$ angles with respect to the disk normal, each of them corresponds to definite direction of the mode rotation. The measured gyrotron output power (up to $2 \mathrm{MW}$ ), interaction efficiency (34\%) and diffraction losses in the mode converter $(<2 \%)$ agree well with the design values. The new gyrotron scheme alloys principal enhancement in the device parameters - possibility of electronic switching of output wave beam direction by a cathode coil, frequency stabilization by reflection, possibility to arrange an effective scheme to input signal and to provide frequency/phase locking of a gyrotron-oscillator. The latter advance gives very promising perspectives in development of megawatt gyrotrons with higher frequency (higher operating mode) stabilized by an external signal, possibility to make several gyrotrons coherent and combine their power. The first experiments on gyrotron frequency stabilization by optimum reflection (A. Kuftin, A. Chirkov, G. Denisov, 2016) are very encouraging. For rather high voltage variation of 95-100 $\mathrm{kV}$ frequency of the $2 \mathrm{MW}$ gyrotron was stable within $2 \mathrm{MHz}$. Frequency stabilization of moderate power $263 \mathrm{GHz}$ gyrotron by PLL resulted in ultra-narrow (record) oscillation spectrum width as $1 \mathrm{~Hz}[16]$.

Detail simulations and preliminary experiments were made for the scheme of $30 \mathrm{GHz}$ gyro-TWT with nonlinear absorber in a feedback loop. For the second harmonic operation with $68 \mathrm{~kW} / 10$ A electron beam the output radiation of the device consists of periodic pulses with $400 \mathrm{~kW}$ power and $0.2 \mathrm{~ns}$ pulse duration (corresponding frequency bandwidth of $5 \mathrm{GHz}$ ) with $7 \mathrm{~ns}$ interval between pulses. The non-linear absorber based on cyclotron absorption at second harmonic was successfully tested.

Acknowledgements. This discussion on gyrotron development trends would be unthinkable without the work results of my colleagues and collaborators whose group is fortunately large in number.

\section{References}

1. M.K. Thumm, "State-of-the-Art of High Power GyroDevices and Free Electron Masers", KIT Scientific Publishing, 2015.
2. A.V. Krasilnikov, et al. Progress with the ITER Project Activity in Russia. Nuclear Fusion, 2015, 55, \#10, 104007 (11 p).

3. G.G. Denisov, A.G. Litvak, A.V. Chirkov, et al. Development Status of Gyrotron Setup for ITER ECW System. Proceedings of IRMMW THz Conference, Hong Kong, August, 2015.

4. http://www.iterrf.ru/

5. L. Popov, M. Agapova, Yu. Belov et al. Super-High Power Gyrotrons for Electron-Cyclotron Plasma Heating. This conference.

6. Proceedings of $26^{\text {th }}$ IAEA Fusion Energy Conf., Kyoto, Japan, October 2016, Conf. ID: 48315 (CN-234).

7. S.V. Samsonov, I.G. Gachev, G.G. Denisov, et al. "Kaband Gyrotron Traveling-Wave Tubes with the Highest Continuous-Wave and Average Power", IEEE Trans. on Electron Devices, 2014, vol. 61, no.12, p. 4264-4268.

8. S.V. Samsonov, A.A. Bogdashov, G.G. Denisov, et al. Proof-of-Principle Experiment on High-Power Gyrotron Traveling-Wave Tube with a Microwave System for Driving and Extracting Power Through One Window. IEEE Microwave and Wireless Components Letters, 2016, vol.26, no.4, p. 288290.

9. S.V. Samsonov, A.A. Bogdashov, G.G. Denisov, et al. "Recent experiments and simulations on gyro-TWTs with helically corrugated waveguides", Proc. of 41 st Int. Conf. on Infrared, Millimeter and Terahertz Waves, Copenhagen, Denmark, p. H4B5, Sept. 2016.

10. S.V.Samsonov, G.G.Denisov, I.G.Gachev et al. Development of Gyrotron Traveling-Wave Tubes at IAP and GYCOM. This Conference.

11. Procedings of $4^{\text {th }}$ IAEA DEMO Programme Workshop. 14-18 November, 2016, Karlsruhe, Germany.

12. A.V. Chirkov, G.G. Denisov and A.N. Kuftin. Perspective gyrotron with mode converter for co and counter-rotation operating modes. // Applied Physics Letters, 106, 263501 (2015); doi: 10.1063/1.4923269.

13. V.L. Bakunin, G.G Denisov, Y.V Novozhilova. Zones of Frequency Locking by an External Signal in a Multimode Gyrotron of a Megawatt Power Level. Radiophysics and Quantum Electronics, 2016, 58 (12), 893-904.

14. Yu.V.Novozhilova, V.L.Bakunin, A.V.Chirkov et al. Influence of Mode Competition and External Wave Frequency Modulation on Gyrotron Frequency Locking. This Conference.

15. M.Yu. Glyavin, G.G. Denisov, M.L. Kulygin, Yu.V. Novozhilova. Stabilization of gyrotron frequency by reflection from nonresonant and resonant load. Technical Physics Lett., July 2015, V. 41, Issue 7, p. 628-631.

16. G.G. Denisov, A.P. Fokin, M.Yu. Glyavin, et al. High precision frequency stabilization of a $100 \mathrm{~W} / 263 \mathrm{GHz}$ continuous wave gyrotron. Proceedings of $18^{\text {th }}$ Int. Vacuum Elecronics Conf., London, 24-26 April, 2017.

17. N.S. Ginzburg, G.G. Denisov, M.N. Vilkov, et al. Generation of "gigantic" ultra-short microwave pulses based on passive mode-locking effect in electron oscillators with saturable absorber in the feedback loop. Physics of Plasmas, 2016, 23 (5), 050702 . 